\title{
EDUCACIÓN
}

\section{El pensamiento crítico en la formación profesional: indicadores para el desarrollo de las habilidades cognitivas del pensamiento crítico en la Facultad de Filosofía de la Universidad Nacional de Asunción}

\author{
Marta Isabel Canese de Estigarribia', Selva Chávez', Sergio Cáceres \\ Mercado1, Ricardo Estigarribia Velázquez², Gustavo Ibarra², Francisco \\ Bogado², Christian Lugo ${ }^{3}$, Rocío Valenzuela ${ }^{1}$
}

\begin{abstract}
Resumen
Introducción: Este informe de investigación presenta los resultados parciales del proyecto de investigación que tiene por objetivo la elaboración de una guía de indicadores para el desarrollo de las habilidades cognitivas del pensamiento crítico en la Facultad de Filosofía de la UNA.
\end{abstract}

Objetivo: Elaborar una guía de indicadores para la planificación, el desarrollo y la evaluación de las habilidades cognitivas del Pensamiento Crítico en el proceso de formación de profesionales de las carreras impartidas por la Facultad de Filosofía - UNA.

Material y Método: La metodología de la investigación tuvo un enfoque participativo, abordaje mixto, cualitativo y cuantitativo, diseño observacional, alcance descriptivo y corte transversal. El universo del estudio comprende a los estudiantes y profesores de la Facultad de Filosofía de la UNA. En la primera etapa del estudio, el muestreo fue de tipo no probabilístico, y se aplicaron las técnicas: taller participativo, grupo focal y cuestionario. En la segunda etapa, se aplica el instrumento de evaluación del desarrollo de habilidades cognitivas del pensamiento crítico diseñado con los datos de la primera etapa, a una muestra de estudiantes de los últimos cursos de cada carrera de la misma facultad.

Resultados: A partir del análisis de los datos obtenidos, se han identificado las siete habilidades cognitivas consideradas prioritarias por los participantes:

\footnotetext{
1. Universidad Nacional de Asunción.

2. Comisión Nacional de Derechos Humanos (CONADEH).

3. Centro de Investigaciones en Filosofía y Ciencias Humanas.
}

Este reporte de investigación presenta los avances del proyecto titulado "El pensamiento Crítico en la Formación Profesional: indicadores para el desarrollo de las habilidades cognitivas del Pensamiento Crítico en la Facultad de Filosofía de la Universidad Nacional de Asunción", que se realiza en la Facultad de Filosofía (UNA), en vinculación con la Comisión Nacional de Derechos Humanos (CONADEH), y el Centro de Investigaciones en Filosofía y Ciencias Humanas (CIF), el marco del programa PROCIENCIA del Consejo Nacional de Ciencia y Tecnología (CONACYT), de Paraguay.

E-mail: mcanese@gmail.com

DOI: $10.26885 /$ rcei.foro.2018.44 


\section{El pensamiento crítico en la formación profesional. Canese et al.}

interpretación, clarificación, análisis, evaluación, empatía intelectual, solución de problemas, visión transformadora. Los indicadores señalados por los participantes fueron analizados en relación a las teorías, declaraciones y modelos de medición de las habilidades del pensamiento crítico revisados en este estudio, para conformar una guía para la planificación, el desarrollo y la evaluación de las habilidades cognitivas del pensamiento crítico.

Conclusiones: Con la construcción de esta guía de indicadores para el desarrollo de las habilidades cognitivas del pensamiento crítico, la Facultad de Filosofía cuenta con un primer instrumento para el desarrollo de las habilidades cognitivas superiores, que podrá servir de referencia para la mejora de los planes de estudio, las estrategias de enseñanza y los sistemas de evaluación.

Palabras clave: habilidades cognitivas, pensamiento crítico, indicadores.

\section{Referencias}

Ennis, R. (2013). The Nature of Critical Thinking: Outlines of General Critical Thinking Dispositions and Abilities. Illinois: Critical Thinking.

Freire, P. (1987). Pedagogía de la liberación. São Paulo: Editora Moraes.

Ossa-Cornejo, C. J., Palma-Luengo, Maritza R., Lagos-San Martín, N. G., QuintanaBello, I. M., \& Díaz-Larenas, C. H. (2017). Análisis de instrumentos de medición del pensamiento crítico. Ciencias Psicológicas, 11(1), 19-28.

Paul, R., \& Elder, L. (2005). Estándares de competencia para el pensamiento crítico. Estándares, Principios, Desempeño, Indicadores y Resultados. Con una Rúbrica Maestra en el Pensamiento Crítico.

Silvero, J. M., \& Cáceres Mercado, S. (2011). Pensamiento paraguayo. Asunción: CCJZ. 